\title{
Experimental Analysis and Modeling of Temperature Dependence of Lithium-Ion Battery Direct Current Resistance for Power Capability Prediction
}

\author{
Linfeng Zheng ${ }^{1,2}$, Jianguo Zhu ${ }^{1}$, Guoxiu Wang ${ }^{2}$, Dylan Dah-Chuan $\mathrm{Lu}^{1}$, Peter McLean ${ }^{1}$, and Tingting He ${ }^{1}$ \\ ${ }^{1}$ Faculty of Engineering and Information Technology, University of Technology Sydney, Australia \\ ${ }^{2}$ Centre for Clean Energy Technology, University of Technology Sydney, Australia \\ Email: Linfeng.Zheng@student.uts.edu.au
}

\begin{abstract}
Accurate lithium-ion battery power capability prediction gives an indication for managing power flows in or out of batteries within the safe operating area, which is one of the primary challenging functions of battery management systems (BMSs). The battery direct current resistance (DCR) is typically employed for power capability prediction, but its characteristic depends significantly on the ambient temperature. It is essential to investigate systematically the temperature dependence of battery DCR for achieving reliable power capability prediction. Based on a large amount of battery test data, a battery DCR model is proposed for quantitatively describing its temperature dependence. This model is then applied for battery power capability prediction, and the results are verified by experimental results.
\end{abstract}

Index Terms-Battery power capability prediction, battery management system (BMS), battery direct current resistance, temperature dependence.

\section{INTRODUCTION}

As significant alternatives to traditional fossil fuels, lithium-ion batteries always exhibit favorable characteristics, such as high energy and power densities, excellent cycling performance, and environmental friendliness, and therefore have been widely applied in electrical energy storage systems for electric vehicles, smart grids, renewable energy regeneration systems [1], [2], etc. For reliable and safe operation and control of battery systems, battery management systems (BMSs) are indispensable to be deployed with the batteries. One of the key functions of BMSs is to predict the battery power capability for absorbing or delivering a certain power in the safe operating area. However, accurate battery power capability prediction still poses a significant challenge due to the time-variable and nonlinear battery characteristics as well as the influences by operating conditions.

The reported methods for battery power capability prediction can be mainly divided into three groups: model based methods, machine learning methods, and characteristic map based methods. Due to their real-time performance and high adaptation capabilities, the model based methods have predominated in the research of battery power capability prediction approaches [3]-[7], but the complexity of the algorithms of model based methods requiring high computation efforts would be strikingly problematic when implementing in BMSs.

The machine learning methods, such as support vector machine [8] and neural network [9], [10], have the ability to learn and approximate the relationships between the input signals and the output signal (i.e. battery power capability) without being explicitly modeled. However, they treat the battery as a "black-box" system and require a great deal of reliable data to train the relationship between the power capability and the input variables, and the accuracy of the machine learning methods is highly dependent on the training data [11].

Alternatively, the characteristic map based methods utilize the known static dependencies of battery states and parameters, such as SOCs and temperatures, to build a multi-dimensional power performance map [7], which can be directly embedded into BMSs for on-board battery power capability prediction. The battery direct current resistance (DCR) is typically employed to determine the operating point of the characteristic map [12]. It is worth mentioning that the battery DCR characteristic depends significantly on the temperature, and therefore it incurs a time-consuming and tedious battery test process to establish the characteristic map covering a broad temperature range. Another problem is that a high amount of memory capacity is inevitably required to store the multi-dimensional characteristic map in BMSs [13].

To overcome the aforementioned drawbacks, this work investigates systematically the temperature dependence of the DCR and establish an effective DCR model, which is implemented on battery power capability prediction for achieving reliable results.

The remainder of this paper is organized as follows: Section II illustrates the experimental analysis and modeling of the temperature dependence of battery DCR, and then it describes the implementation of the proposed DCR model on battery power capability prediction. The verified results and evaluations of the proposed DCR model and power capability prediction are demonstrated in Section III, followed by the conclusions and future work summarized in Section IV. 


\section{BATTERY DIRECT CURRENT RESISTANCE}

\section{A. Experimental Analysis}

To investigate the temperature dependence of battery DCR and power capability, a battery current-pulse test schedule was specially designed to identify the DCR at different state of charges (SOCs) under various ambient temperatures. The current-pulse tests were conducted from $10{ }^{\circ} \mathrm{C}$ to $40{ }^{\circ} \mathrm{C}$ at an interval of $5{ }^{\circ} \mathrm{C}$. In each current-pulse test, the battery was charged with $\mathrm{C} / 3$ at every $5 \%$ SOC increment. The DCR was then determined by the current pulse $(\Delta I)$ and its resulting voltage response $(\Delta V)$, as expressed by

$$
R=\Delta V / \Delta I
$$

where $R$ is the battery DCR.

For excluding the impacts of the changing open circuit voltage (OCV) and the diffusion process on the measurement of battery DCR, the voltage change between $1 \mathrm{~s}$ after the start of the current pulse is applied for the DCR calculation. Fig. 1 depicts battery charge DCRs at different SOCs under various ambient temperatures. As can be observed, the temperature has a significant impact on battery DCR, which increases with the decreasing temperature. For example, at 5\% SOC, the battery DCR is about $2.673 \mathrm{~m} \Omega$ under the temperature of $10{ }^{\circ} \mathrm{C}$, which approximates 2.85 times of that under the temperature of $40{ }^{\circ} \mathrm{C}$ (i.e. $0.938 \mathrm{~m} \Omega$ ). It is noted that the DCRs difference between two adjacent temperatures strikingly decreases with the increasing temperature. However, the relationship between the DCR and temperature still could not be quantitively extracted from Fig. 1, and needs to further investigated.

The linear representation of battery DCR is changed to the logarithmic representation, and the logarithmic DCRs are demonstrated in Fig. 2. It can be observed that the logarithmic DCRs appear a similarly decreasing tendency with the increasing temperature for all different cell SOCs. It is noteworthy mentioning that the logarithmic DCR versus temperature curves for various battery SOCs are approximately parallel to each other. This characteristic is of major importance to estiblish a model to describe the relationship between the temperature and battery DCR, which would be elaborated in the following section.

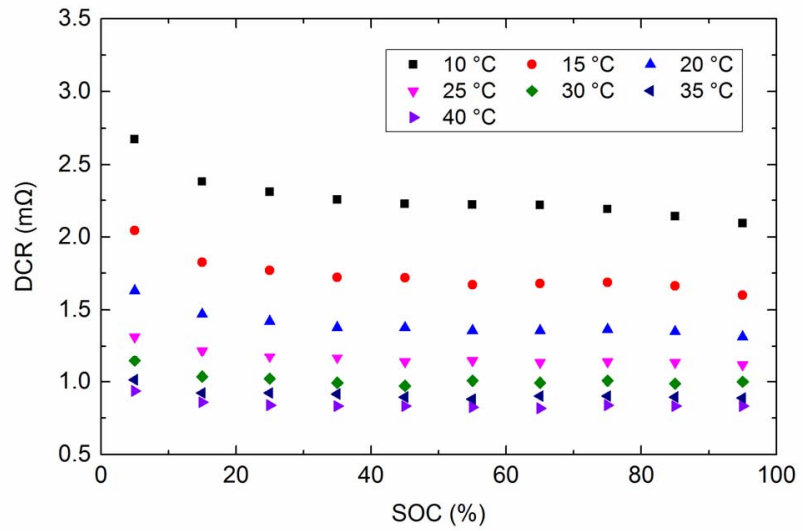

Fig. 1. Battery DCRs at different SOCs and temperatures.

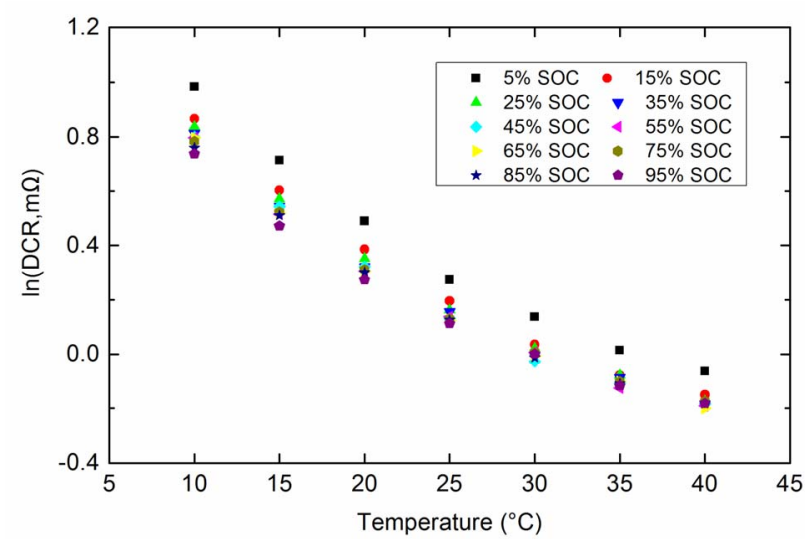

Fig. 2. Battery logarithmic DCRs at different SOCs and temperatures.

\section{B. Modeling of DCR}

The relationship between the logarithmic DCR and temperature can be quantitatively expressed as a set of quadratic equations with the same rate of increase/decrease for different SOCs. Thus, at various battery SOCs, the temperature-dependent DCRs are modeled as

$$
\ln (R(T, S O C))=\alpha T^{2}+\beta T+\gamma(S O C)
$$

where $R(T, S O C)$ denotes the DCR under the temperature $T ; \alpha$ and $\beta$ are two constant parameters for different SOCs; and $\gamma(S O C)$ denotes the intercept of the equation at the certain SOC.If one temperature is considered as the based temperature, its corresponding DCR, also called the based DCR in the presented work, is governed by

$$
\ln \left(R_{b a s}(S O C)\right)=\alpha T_{b a s}^{2}+\beta T_{b a s}+\gamma(S O C)
$$

where $R_{b a s}(S O C)$ and $T_{\text {bas }}$ denote the based DCR and temperature, respectively.

By combining (2) and (3), one has

$$
\ln \left(\frac{R(T, S O C)}{R_{b a s}(S O C)}\right)=\left(\alpha T^{2}+\beta T\right)-\left(\alpha T_{b a s}^{2}+\beta T_{b a s}\right)
$$

It is worth mentioning that the SOC dependent parameter $\gamma(S O C)$ is counteracted in (4), and $\alpha$ and $\beta$ are constants for different SOCs. With some arrangements, Equation (4) can be rewritten as

$$
\begin{aligned}
R(T, S O C) & =R_{b a s}(S O C) \\
& \cdot \exp \left[\alpha\left(T^{2}-T_{b a s}^{2}\right)+\beta\left(T-T_{b a s}\right)\right]
\end{aligned}
$$

In this paper, the room temperature (i.e. $25^{\circ} \mathrm{C}$ ) and its corresponding DCRs at different SOCs are regarded as the based temperature and DCRs. Thus, with the based values, Equation (5) can be used to compute battery DCRs at different SOCs and temperatures.

\section{Power Capability Prediction}

A simple battery model consisting of an OCV and a resistance (i.e. DCR) is widely used for computing battery peak loading current within the permitted voltage limit [14]-[16]. The approximate relationship between the 
loading current and cell terminal voltage is governed by

$$
U=O C V-R(T, S O C) \times I
$$

where $U$ and $I$ denote the battery terminal voltage and loading current, respectively.

The battery peak charge current is then given by

$$
I_{\max }=\left(U_{\max }-O C V\right) / R(T, S O C)
$$

where $I_{\max }$ denotes the peak charge current, and $U_{\max }$ denotes the permitted maximum cell terminal voltage.

Then, battery charge power capability is determined by

$$
P_{\max }=U_{\text {max }}\left(U_{\text {max }}-O C V\right) / R(T, S O C)
$$

where $P_{\max }$ denotes the battery charge power capability.

\section{VERIFICATIONS AND DISCUSSIONS}

The mean absolute percentage error (MAPE) is employed to evaluate the performance of the proposed methods for predicting battery DCRs and power capabilities under various ambient temperatures. The MAPE gives an indication of how accurate a forecasting method is in statistics, and it is governed by

$$
M A P E=\frac{1}{n} \sum_{i=1}^{n}\left|\left(f_{i}-y_{i}\right) / y_{i}\right| \times 100 \%
$$

where $f_{i}$ and $y_{i}$ denote the predictive and referenced values, respectively, and $n$ denotes the number of data set of predictive and referenced values.

\section{A. DCR Prediction}

The parameters of the DCR model were fit by using the Levenberg-Marquardt algorithm [17], [18], and are listed in Table I. The coefficient of determination $R^{2}$ is about 0.9972 , which means that the proposed model can precisely match the temperature dependence of battery DCR.

Fig. 3 shows the results of battery DCR prediction at different SOCs and temperatures, in which Ref. and Pre. denote the referenced and predictive values, respectively. It can be seen that under various ambient temperatures, the predictive battery charge DCRs can follow the tracks of the referenced values at different SOCs. Since the DCRs under $25{ }^{\circ} \mathrm{C}$ are selected as the based values, the forecasts computed by the DCR model are entirely identical to the referenced values.

The MAPEs of the DCR predictions are plotted in Fig. 4 , where the maximum charge DCR MAPE is $1.80 \%$ occurred at the temperature of $15{ }^{\circ} \mathrm{C}$. It suggests that the proposed DCR model is able to describe the temperature dependence of both battery DCRs well and estimate the DCR values with high accuracy, which provides a credible foundation for subsequent predictions of battery power capabilities.

TABLE I

Optimal Parameters OF THE Proposed DCR MODEL

\begin{tabular}{|c|c|}
\hline Parameter & Coefficient \\
\hline$\alpha$ & 0.0007545 \\
\hline$\beta$ & -0.07033 \\
\hline$R^{2}$ & 0.9972 \\
\hline
\end{tabular}

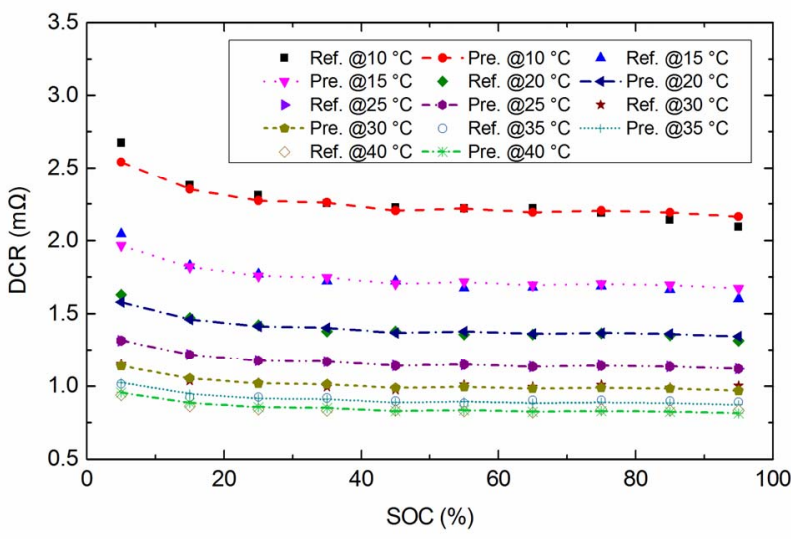

Fig. 3. Battery DCR prediction results at different SOCs and temperatures.

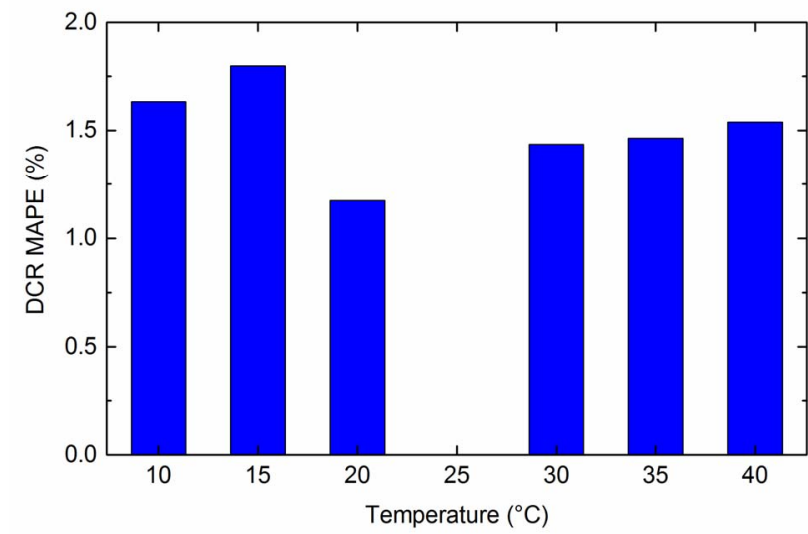

Fig. 4. MAPEs of battery DCR prediction under various temperatures.

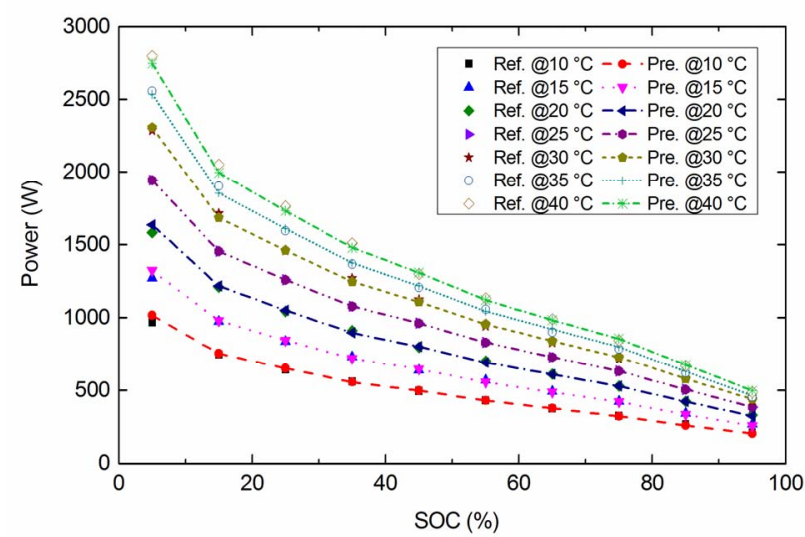

Fig. 5. Battery power capability prediction results at different SOCs and temperatures

\section{B. Power Capability Prediction}

The battery DCR forecasts and Equation (8) are applied to predict battery power capabilities, and the results are shown in Fig. 5, where Ref. and Pre. denote the referenced powers the predictive values, respectively.

Since the mentioned-above DCR forecasts can track well with the referenced values, the predictive battery charge power capabilities can effectively follow the referenced values with small errors at different SOCs and temperatures, as shown in Fig. 5. Their MAPEs are depicted in Fig. 6. Strikingly, all MAPEs are less than 2\% 


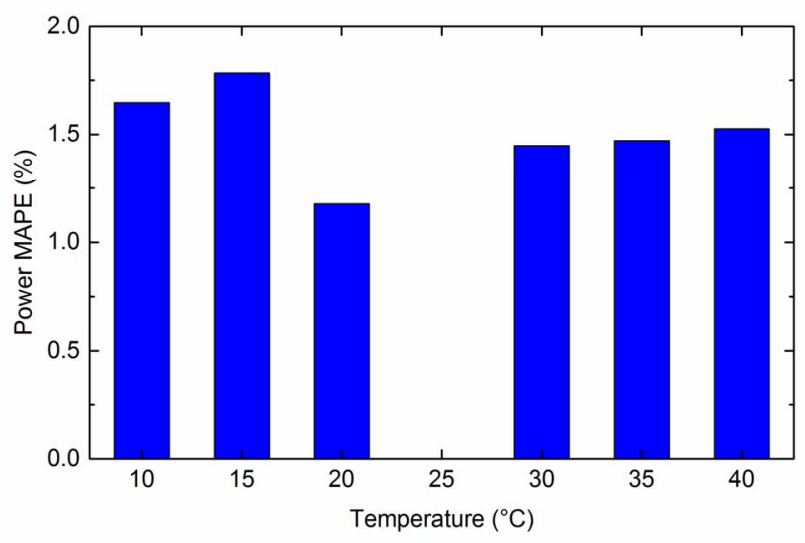

Fig. 6. MAPEs of battery power capability prediction under various temperatures.

under different ambient temperatures, which are similar to the MAPEs of the DCR prediction. It indicates that the proposed method can effectively forecast battery charge power capability under different battery operating temperatures.

\section{CONCLUSIONS}

This paper proposed a novel battery direct current resistance (DCR) model for quantitatively describing its temperature dependence and incorporated the model for predicting battery power capability. On the basis of the experimental analysis of battery test data, the logarithmic DCRs could be approximately expressed as a set of quadratic equations for various ambient temperatures. With the based values, the temperature-dependent DCR was further modeled as an exponential function. The DCR model was then employed for battery charge power capability prediction. The experimental results indicated that the proposed approaches can achieve high accuracy for both battery charge DCR and power capability predictions. The proposed temperature-dependent DCR model is promising to be implemented in BMSs for battery power capability prediction, which would lighten the memory-consuming of BMSs in comparison with the conventional characteristic map based methods. The discharge DCR model and its implementation on battery discharge power prediction will be investigated in our future work.

\section{ACKNOWLEDGMENT}

This work was supported by Rail Manufacturing Cooperative Research Centre (RMCRC).

\section{REFERENCES}

[1] J. Cho, S. Jeong and Y. Kim, "Commercial and research battery technologies for electrical energy storage applications," Progress in Energy and Combustion Science, vol. 48, pp. 84-101, June 2015.

[2] L. Zheng et al, "Novel methods for estimating lithium-ion battery state of energy and maximum available energy," Appl. Energy, vol. 178, pp. 1-8, September 2016.

[3] W. Zhang, W. Shi and Z. Ma, "Adaptive unscented Kalman filter based state of energy and power capability estimation approach for lithium-ion battery," J. Power Sources, vol. 289, pp. 50-62, September 2015.

[4] Z. Wei et al, "An adaptive model for vanadium redox flow battery and its application for online peak power estimation," J. Power Sources, vol. 344, pp. 195-207, March 2017.

[5] F. Sun, R. Xiong and H. He, "Estimation of state-of-charge and state-of-power capability of lithium-ion battery considering varying health conditions," J. Power Sources, vol. 259, pp. 166-176, August 2014.

[6] R. Xiong et al, "Model-based state of charge and peak power capability joint estimation of lithium-ion battery in plug-in hybrid electric vehicles," J. Power Sources, vol. 229, pp. 159-169, May 2013.

[7] A. Farmann and D. U. Sauer, "A comprehensive review of on-board State-of-Available-Power prediction techniques for lithium-ion batteries in electric vehicles," J. Power Sources, vol. 329, pp. 123-137, October 2016.

[8] F. Zheng et al, "Temperature dependent power capability estimation of lithium-ion batteries for hybrid electric vehicles," Energy, vol. 113, pp. 64-75, October 2016.

[9] C. Fleischer et al, "Adaptive On-line State-of-availablepower Prediction of Lithium-ion Batteries," Journal of Power Electronics, vol. 13, pp. 516-527, 2013.

[10] W. Haiying et al, "Power state prediction of battery based on BP neural network," Proc. of 7th Int. Forum On Strategic Technology (IFOST), Tomsk (Russia), Nov. 2012, pp. 1-4.

[11] L. Zheng et al, "Co-estimation of state-of-charge, capacity and resistance for lithium-ion batteries based on a highfidelity electrochemical model," Appl. Energy, vol. 180, pp. 424-434, October 2016.

[12] W. Waag, S. Käbitz and D. U. Sauer, "Experimental investigation of the lithium-ion battery impedance characteristic at various conditions and aging states and its influence on the application," Appl. Energy, vol. 102, pp. 885-897, February 2013.

[13] M. Sayegh et al, "Temperature dependency modelling of LFP/C battery for an aeronautical application," Proc. of 2016 Int. Conf. on Electrical Systems for Aircraft, Railway, Ship Propulsion and Road Vehicles \& International Transportation Electrification Conference (ESARS-ITEC), Toulouse (France), Nov. 2016, pp. 1-7.

[14] J. R. Belt, "Battery Test Manual for Plug-in Hybrid Electric Vehicles," Idaho National Laboratory, INL/EXT-07-12536, Dec. 2010.

[15] G. L. Plett, "High-performance battery-pack power estimation using a dynamic cell model," IEEE Trans. Veh. Technol., vol. 53, pp. 1586-1593, September 2004.

[16] C. Zhang, C. Zhang and S. Sharkh, "Estimation of real-time peak power capability of a traction battery pack used in an HEV," Proc. of 2010 Asia-Pacific Power and Energy Engineering Conf. (APPEEC), Chengdu (China), March 2010, pp. 1-6.

[17] K. Levenberg, "A method for the solution of certain nonlinear problems in least squares," Q Appl Math, vol. 2, pp. 164-168, 1944.

[18] J. J. Moré, "The levenberg-marquardt algorithm: Implementation and theory," Numerical Analysis, G. A. Watson, Ed. New York: Springer-Verlag, pp. 105-116, 1978. 